\title{
Test of Time Reversal Invariance at the COSY storage ring
}

Yury Valdau*

Institut für Kernphysik, Forschungszentrum Jülich, 52425 Jülich, Germany

Helmholtz-Institut für Strahlen- und Kernphysik Nussallee 14-16, 53115 Bonn, Germany

E-mail: y.valdauefz-juelich.de

Time-reversal symmetry is one of the most fundamental symmetries in nature. CP-violating phenomena, which can be regarded as equivalent to T-violation on the basis of the CPT theorem, have been observed in the $K^{0}$ and $B$ systems. The current upper limit for time-reversal (parity conserving) non-invariance was obtained through measurements of the total cross sections of a polarized neutron beam incident on a Holmium target. However, any understanding of these measurements at a fundamental level is difficult due to the complex nature of the nuclear target. The theoretical interpretation of measurements with a polarized proton beam and a deuterium target would be much cleaner. Measurements of such a kind were proposed to the COSY-PAC and first tests have been done. The status of this experiment, as well as plans for improvements, are here presented.

8th International Conference on Nuclear Physics at Storage Rings-Stori11,

October 9-14, 2011

INFN, Laboratori Nazionali di Frascati, Italy

* Speaker. 
The CPT theorem links together all three fundamental symmetries of nature. If one assumes that $\mathrm{CPT}$ invariance is valid, it means that $\mathrm{CP}$ violation must be compensated by $\mathrm{T}$ violation. The charge $\mathrm{C}$ and parity $\mathrm{P}$ symmetries, are both broken by the weak interaction. $\mathrm{CP}$ violation has been studied in the $K^{0}$ and $B$ systems, but the strength determined here seems to be insufficient to explain the apparent difference between matter and antimatter in the Universe, and hence our very existence. It is therefore very important to study discrete symmetries to understand more about the origin of the symmetry breaking.

The most established and precise test of $\mathrm{CP}$ violation is the search for a permanent electric moment (EDM) of an elementary particle [1]. However, any such a discovery would mean the simultaneous breaking of P-parity and T-time symmetries and thus, in order to get information about the breaking of time symmetry, one has to use a theoretical model [2]. In this contribution, a T-even P-odd test of T-invariance is discussed which, in conjunction with EDM measurements, might help one to understand better the origin of symmetry breaking.

\section{Current status of TRI tests}

P-even TRI studies are usually detailed balance or P-A tests [3]. In such an experiment two independently measured observable are compared, which is why the accuracy is limited to $\approx 10^{-3}$. Other tests of T-invariance involve studying the decays of nuclei or $K^{0}$ mesons or the scattering of neutrons from nuclei [4]. The current upper limit for time-reversal (parity conserving) noninvariance was obtained through the measurement of the total cross section of a polarized neutron beam incident on a Holmium target [5]. However, any interpretation of measurements carried out with a complex nuclear target requires extensive modeling, which leads to some model dependence in the extracted upper limit.

In order to improve the accuracy in the upper limit obtained from a scattering process involving elementary particles by several orders of magnitude, it is necessary to perform a null experiment. Here one single observable should be measured to a very high precision. Deviation from zero for the selected observable would then indicate a violation of the symmetry being tested.

It was proven quite generally that there exists no null experiment to test time-invariance in a reaction with two particles in and two out [6]. However, this theorem does not apply to studies of total cross sections and it has been shown that measurements of the total cross section asymmetry $A_{y, x z}$ of vector polarized spin-half particles interacting with tensor polarized spin-one particles, would be a true null test of TRI [7]. The principle of the measurements is illustrated in Fig. 1 and discussed extensively in Ref. [8]. More detailed analysis of this observable has shown that the determination of $A_{y, x z}$ in proton-deuteron collisions to $10^{-6}$ would allow to improve the upper limit on time-reversal invariance by an oder of magnitude [9].

\section{Test of Time-Reversal Invariance at COSY}

At the COoler-SYnchrotron COSY-Jülich, a vector polarized proton beam together with an internal tensor polarized deuteron target can be used in the measurement of the $A_{y, x z}$ dependence of the total cross section [8]. COSY will be here used as both an accelerator and ideal zero degree spectrometer. In order to determine the spin-dependent total cross section one needs to measure 


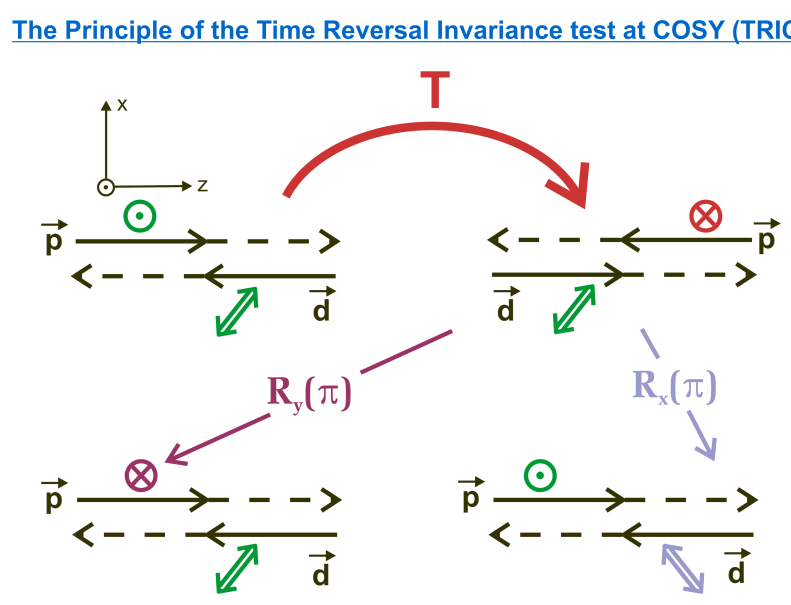

Figure 1: Principle of the TRIC experiment. A vector polarized beam of spin-half particles interacts with a tensor polarized spin-one target. (Picture is from Ref. [8])

the beam current loss as a function of time and then utilize the optical theorem to evaluate the total cross section. This requires:

- A high intensity proton beam and thick polarized gas target.

- Long and stable beam lifetimes.

- Measurements of the beam current with a precision of $10^{-6}$.

\section{Feasibility test}

The first beam time for a proof-of-principle experiment and the measurement of $p p$ total cross sections was taken in 2004. A detailed discussion of the results obtained can be found in Ref. [11]. Three main conclusions were drawn:

- A measurement of the total cross section using a beam-current transformer is possible.

- The Barghausen noise from the ferromagnetic core of the BCT does not allow one to reach the desired precision in the existing measurement.

- Both beam intensity and target thickness must be significantly increased to reach the required sensitivity to test time reversal invariance.

\section{Possible improvements}

There has been significant experience in recent years in the preparation of polarized beams for internal and external COSY experiments. The continuous injection of a beam into the storage ring increases the beam intensity and makes it possible to have experiments with a storage-cell target at the ANKE or PAX experimental positions in the ring. Furthermore, the COSY low beta section, 
the atomic beam source, and the openable storage-cell, which is now available at the PAX station, allow one to get much higher luminosities than before [12]. Therefore it is time to update the initial TRIC proposal to reflect the current state of the art in this area.

In addition to higher polarized beam intensity and thicker polarized gas, there have also been some developments in the technology of beam-current measurements. The design resolution currently available from the Bergoz New Parametric Current Comparator (NPCT) [13] is a factor of two better than that of the BCT used at COSY. Furthermore, a new type of beam current transformer is being developed for the FAIR injection lines. This uses SQUIDs for the readout of the tiny magnetic field from the toroid around the beam pipe [14]. A prototype of such a beam-measurement device has been successfully tested and has shown a resolution of $0.25 \mathrm{nA}$.

Another approach might be to perform a TRI experiment with a bunched beam. In this case, instead of using an active current transformer that is appropriate for measurements of the DC currents in an accelerator, one could employ an Integrating Current Comparator, which allows one to reach a resolution of the order of $1 \mathrm{nA}$ [15]. It was shown that it is possible to measure the intensity of a stored beam to the level of $0.1 \mathrm{nA}$ with this technique [16].

Due to development in electronics, the readout scheme of the TRIC experiment can also be improved. Fast 24 bit ADC can be used instead of the voltage-to-frequency converter (VFT) assumed in the initial design. This would lead to an improvement in the resolution in the beam current measurement by a factor of ten. The replacement of the VFT by a fast high resolution ADC, together with additional improvements in the time measurement, would result in a factor of ten better precision in the beam current readout system.

\section{Conclusions}

Much experience in the operation of polarized beam and targets has been gained at COSY since the initial TRI tests were performed. The low beta section, together with the openable storage cell, available at the PAX position, allow one to reach luminosities sufficient to perform doublepolarized experiments and, in particular, to test time-reversal invariance at COSY.

\section{References}

[1] F. Rathmann, Precursor experiments to search for permanent electric dipole moments (EDMs) of protons and deuterons at COSY, these proceeding.

[2] A. Kurylov, G. C. McLaughlin, and M. J. Ramsey-Musolf, Constraints on T-odd, P-even interactions from electric dipole moments, reexamined, Phys. Rev. D 63 (2001) 076007.

[3] T. S. Bhatia et al., Difference between Polarization and Analyzing Power for 800-MeV n-p Elastic Scattering: Test of Time-Reversal Invariance, Phys. Rev. Lett. 48 (1982) 227.

[4] A. G. Beda and V. R. Skoy, Current Status of Research on T Invariance in Neutron Nuclear Reactions, Physics of Particles and Nuclei 38 (2007) 775.

[5] P. R. Huffman et al., Test of parity-conserving time-reversal invariance using polarized neutrons and nuclear spin aligned holmium, Phys. Rev. C 55 (1997) 2684.

[6] F. Arash, M.J. Moravcsik, and G.R. Goldstein, Dynamic Independent Null Experiment for Testing Time-Reversal Invariance, Phys. Rev. Lett. 54 (1985) 2649. 
[7] H.E. Conzett, Null test of time-reversal invariance, Phys. Rev. C 48 (1993) 423.

[8] P.D. Eversheim, Test of Time-Reversal Invariance in Proton-Deuteron Scattering, COSY Proposal \#22 (1994).

[9] M. Beyer, Test of time-reversal symmetry in the proton-deuteron system, Nucl. Phys. A $\mathbf{5 6 0}$ (1993) 895.

[10] D. Samuel, Test of Feasibility of a Novel High Precision Test of Time Reversal Invariance, Dissertation, Helmholtz Institute für Strahlen- und Kernphysik, University Bonn (2007)

[11] D. Eversheim et al., The feasibility of the TRIC experiment at COSY, Hyperfine Interact 193 (2009) 335.

[12] D. Oellers for the PAX collaboration, Spin Filtering Experiment at COSY - First results, these proceeding.

[13] Bergoz Instrumentation, New Parametric Current Transformer, User Manual rev 1.0 (2007).

[14] A. Steppke et al., Application of LTS-SQUIDs in nuclear measurement techniques, IEEE Transactions on Applied Superconductivity 19 (2009) 768.

[15] Bergoz Instrumentation, Integrating Current Transformer, User Manual rev 3.0 (2010).

[16] A. Paal et al., Bunched beam current measurements with 100 pA RMS resolution at CRYRING, Proceedings of EPAC2006, Edinburg, Scotland, (2006) 1196. 